\title{
Pediatric cardiac retransplant: Differing patterns of primary graft failure by age at first transplant
}

\author{
John M. Karamichalis, MD, ${ }^{\text {a,b }}$ Shelley D. Miyamoto, MD, ${ }^{\text {a,b }}$ David N. Campbell, MD, ${ }^{\text {a,b }}$ \\ Jilayne Smith, MSN, ${ }^{\text {a,b }}$ Kim K. McFann, PhD, ${ }^{c}$ Sydne Clark, BS, ${ }^{\mathrm{b}}$ Biagio Pietra, MD, ${ }^{\mathrm{a}, \mathrm{b}}$ and \\ Max B. Mitchell, MD ${ }^{\mathrm{a}, \mathrm{b}}$
}

Objective: This study compared graft failure leading to retransplant in infants versus older children at initial heart transplant.

Methods: Twenty-six retransplant recipients were compared by age at first transplant: infant group ( $<1$ year) and pediatric group ( $\geq 1$ year).

\begin{abstract}
Results: Early retransplant survival was $92 \%$. Retransplant survivals at 1,3 , and 5 years were $83 \%, 74 \%$, and $67 \%$. There were 15 infant and 11 pediatric patients. First transplant ages were $0.4 \pm 0.3$ vs. $8.5 \pm 5.7$ years in infant and pediatric groups, respectively $(P<.01)$. First graft rejection episodes were more common in pediatric group $(4.8 \pm 2.5$ vs $3.1 \pm 2.1, P=.032)$, and rejection rate was higher $(1.5 \pm 1.1$ vs $0.4 \pm 0.4, P=.0024)$. Median first graft survival was longer in infant group (10.7 years vs 3.9 years, $P<.001)$. Recurrent cellular rejection was retransplant indication in $40 \%$ of infant group versus $91 \%$ of pediatric group $(P<.05)$. Cardiac allograft vasculopathy was more prevalent in infant group (73\% vs $20 \%$ in pediatric group, $P=.032$ ).
\end{abstract}

Conclusions: Infant heart transplant recipients had longer primary graft survival, fewer cellular rejection episodes, and higher incidence of cardiac allograft vasculopathy relative to older graft recipients requiring retransplant. Advantages in adaptive immunity in infant heart recipients confer improved primary graft survival, but longer graft life in these patients is limited by cardiac allograft vasculopathy. Older recipient first graft failure was rejection related, and shorter graft life probably limited development of cardiac allograft vasculopathy. (J Thorac Cardiovasc Surg 2011;141:223-30)

Transplant is effective treatment for infants and children with end-stage heart failure or congenital heart disease that is not amenable to repair. ${ }^{1-3}$ When graft failure occurs in these patients, retransplant is the only treatment option. ${ }^{4-11}$ Common causes of graft failure leading to retransplant are cardiac allograft vasculopathy (CAV), various forms of rejection, and "nonspecific" graft failure. ${ }^{4-5,7-9,11}$ Previous single-center studies have reported excellent outcomes, with 3-year retransplant survivals equal to primary transplant. ${ }^{5,7,11}$ These studies, however, are limited by small patient numbers and short follow-up. Larger United Network for Organ Sharing (UNOS) and Pediatric Heart Transplant Study (PHTS) registry studies have clearly demonstrated inferior outcomes for repeated transplants relative to primary transplants in children. ${ }^{4,9}$ In addition, the 2009 International Society for Heart and Lung Transplantation

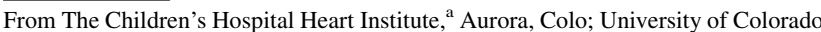
Denver Health Science Center, ${ }^{\mathrm{b}}$ Aurora, Colo; and University of Colorado Denver, ${ }^{\mathrm{c}}$ Colorado School of Public Health, Aurora, Colo.

Disclosures: Authors have nothing to disclose with regard to commercial support.

Read at the 90th Annual Meeting of The American Association for Thoracic Surgery, Toronto, Ontario, Canada, May 1-5, 2010.

Received for publication April 30, 2010; revisions received Aug 5, 2010; accepted for publication Sept 8, 2010; available ahead of print Nov 8, 2010.

Address for reprints: Max B. Mitchell, MD, The Children's Hospital Heart Institute, 13123 E 16th Ave, B-200, Aurora, CO 80045 (E-mail: mitchell.max@tchden.org). $0022-5223 / \$ 36.00$

Copyright (C) 2011 by The American Association for Thoracic Surgery doi:10.1016/j.jtcvs.2010.09.018
}

pediatric registry report now indicates that retransplant is associated with worse 1- and 5-year outcomes. ${ }^{12-13}$

Infant heart recipients have superior long-term outcomes relative to other age groups, with a primary graft half-life of 18 years versus only 11 years for teenagers. ${ }^{12}$ The infant survival advantage is presumably due to the plasticity of their immature immune system, and many centers, including ours, use less immunosuppression in infant recipients to obtain outcomes that exceed those of older children. In reports derived from registry data from UNOS and PHTS, $\mathrm{CAV}$ was the indication for retransplant for approximately half of all patients who underwent primary transplant as children. ${ }^{4,9}$ Given that 3-year graft survival after angiographic diagnosis of CAV in children is only $45 \%$, any angiographic evidence of CAV portends graft failure. ${ }^{12}$ Older recipient and donor ages at first transplant are risk factors for development of CAV, and freedom from CAV with time is longest in infant recipients. ${ }^{12,14}$

Although registry data demonstrate superior primary graft survival for infant heart recipients, this has not been demonstrated for the subset of patients who have undergone retransplant. Reports on pediatric heart retransplant also have not examined the influence of recipient age on the etiology of graft failure leading to retransplant, or determined whether there is any effect on second graft survival. With the known outcome differences for primary transplant between infants and older patients, it is possible that factors leading 


\section{Abbreviations and Acronyms \\ $\mathrm{CAV}=$ cardiac allograft vasculopathy \\ PHTS $=$ Pediatric Heart Transplant Study \\ UNOS $=$ United Network for Organ Sharing}

to first graft failure and retransplant differ by age at initial transplant. The aims of this study were (1) to assess early and midterm outcomes of pediatric heart retransplant at our center, (2) to determine whether primary and second graft survivals differ between patients with initial transplant in infancy and older recipients, and (3) to determine whether age at initial transplant affects primary graft failure etiology and the prevalence of angiographically evident CAV in pediatric patients undergoing retransplant at our center.

\section{MATERIALS AND METHODS Data Collection}

The Colorado Multiple Institutional Review Board approved this study. A waiver of consent was obtained, and patient data were made anonymous. All heart transplants performed at our center between 1990 and March 2010 were retrospectively reviewed. Retransplant patients were identified, and patients were compared by recipient age at initial transplant: infant group ( $<1$ year) and pediatric group ( $\geq 1$ year). The dominant listing diagnoses for first and second transplants were obtained from the original UNOS listing entries. At our center, rejection history, serial biopsy, and selective coronary angiographic results are used to establish a dominant listing diagnosis for patients with graft failure related to mixed rejection and CAV. Classification of graft failure as idiopathic is avoided. Echocardiographic and catheterization data were extracted from the most recent studies performed before retransplant listing. Variables included ages at first and second transplants, donor and recipient cytomegalovirus serologic statuses at each transplant, primary graft rejection episodes, presence and severity of CAV at retransplant listing, first and second graft survivals, early and late retransplant mortalities, retransplant ventilator time, and intensive care unit and hospital stays. Early mortality was considered death before hospital discharge or within 30 days of retransplant if after discharge.

\section{Immunosuppression and Surveillance}

All patients received induction, maintenance immunosuppression, and graft surveillance according to institutional protocol. Perioperative induction included steroids, antithymocyte globulin, intravenous immune globulin, calcineurin-inhibitor (cyclosporine, INN ciclosporin), and azathioprine. Maintenance immunosuppression was steroid free. Infant recipients who were free of rejection were transitioned to single-drug maintenance (cyclosporine) after 1 year. Patients who underwent transplant outside infancy and were free of rejection at 1 year continued dual drug therapy (cyclosporine and azathioprine). Infant graft surveillance was primarily noninvasive and consisted of serial clinical assessments and echocardiograms. ${ }^{15}$ Initial right heart surveillance catheterization with endomyocardial biopsy was performed at 1 year for recipients at least 4 years old, at 3 months for those aged 4 to 8 years, and at 2 months and 6 months for patients older than 8 years. All patients underwent endomyocardial biopsy, hemodynamic assessment, and selective coronary angiography at 1 year after transplant and then at 2-year intervals. Catheterization, biopsy, and coronary evaluations were done as indicated by conflicting clinical and echocardiographic findings or for atypical or complex rejection. More frequent coronary assessment was performed if CAV was diagnosed.
Retransplant management was protocol based. Perioperative induction included steroids, azathioprine, calcineurin-inhibitor (cyclosporine or tacrolimus), intravenous immune globulin, and antithymocyte globulin. Maintenance immunosuppression was dual therapy with calcineurin-inhibitor and mycophenolate mofetil. Target calcineurin-inhibitor levels were higher than with primary transplants. Immunosuppression was adjusted on the basis of renal function and evidence of hypertension. All retransplant recipients received total lymphoid irradiation at 1 posttransplant week.

\section{Rejection}

A rejection episode was considered an event leading to augmentation of immunotherapy, as defined by the PHTS. ${ }^{4,16-18}$ Rejection diagnosis was based on clinical findings, echocardiography, or endomyocardial biopsy. Total rejection episodes and rejection rate per graft year were determined. Rejection was treated with steroids or steroids combined with antilymphocyte antibody (thymoglobulin or OKT3). Use of adjunct therapy for resistant rejection depended on the underlying cause of graft rejection. Treatment of ongoing cellular rejection consisted of total lymphoid irradiation and additional cytotoxic or antimetabolite therapy. Resistant antibody-mediated rejection was treated with plasmapheresis and intravenous immune globulin.

\section{Cardiac Allograft Vasculopathy}

Diagnosis of CAV was based on selective coronary angiography and was classified as none, mild, moderate, or severe according to the Cardiac Transplant Research Database criteria (methodology adopted by the PHTS). ${ }^{14,19}$ All studies were classified by a single independent observer.

\section{Statistical Methods}

Continuous variables were compared with independent sample Student $t$ test. Categoric variables were compared with $\chi^{2}$ test of independence and Fisher's Exact test. Graft survival was estimated by the Kaplan-Meier method, and comparisons were with log-rank test. Poisson regression was used to evaluate the count of rejection episodes. Statistical analysis was performed with the SAS statistical package version 9.2 (SAS Institute Inc, Cary, NC).

\section{RESULTS}

Of 350 pediatric heart transplants, 26 retransplants were performed in 26 patients. No retransplants were for early graft failure. One occurred at 1 year, and the remainder were performed later than 2 years after primary transplant. Etiologies of graft failure were rejection-related graft dysfunction $(\mathrm{n}=16,62 \%), \operatorname{CAV}(\mathrm{n}=7,27 \%)$, and right ventricular failure with severe tricuspid regurgitation $(\mathrm{n}=3,12 \%)$. Five patients with rejection-related failure also had angiographically evident CAV ( 2 mild, 2 moderate, 1 severe), and 1 patient with right ventricular failure also had angiographically evident CAV (mild). All 7 patients with dominant diagnosis of CAV had severe disease. The mean age at primary transplant was $3.9 \pm 5.5$ years. Fifteen patients underwent primary transplant as infants (infant group), and 11 patients were older than 1 year (pediatric group). The infant group represented $8.5 \%$ of all primary infant transplants, and the pediatric group represented $7.4 \%$ of older primary recipients. Mean ages at primary transplant were $0.4 \pm 0.3$ and $8.5 \pm 5.7$ years for infant and pediatric groups, respectively $(P<.01)$. Table 1 presents patient variables at first and second transplants. 
TABLE 1. Patient and group characteristics

\begin{tabular}{|c|c|c|c|c|}
\hline Characteristics & All patients $(n=26)$ & Infant group $(n=15)$ & Pediatric group $(n=11)$ & $P$ value \\
\hline \multicolumn{5}{|l|}{ Primary transplant } \\
\hline Age at transplant $(y$, mean $\pm S D)$ & $3.9 \pm 5.5$ & $0.4 \pm 0.3$ & $8.5 \pm 5.7$ & .0001 \\
\hline Donor age $(y$, mean $\pm S D)$ & $3.9 \pm 11$ & $0.95 \pm 0.66$ & $19.1 \pm 9.0$ & $<.0001$ \\
\hline Listing diagnosis congenital heart disease (no.) & $14(54 \%)$ & $10(66.7 \%)$ & $4(36.4 \%)$ & .23 \\
\hline Listing diagnosis cardiomyopathy (no.) & $12(46 \%)$ & $5(33.3 \%)$ & $7(63.6 \%)$ & .23 \\
\hline Recipient cytomegalovirus seropositive (no.) & $27.3 \%$ & $38.5 \%$ & $11.1 \%$ & .33 \\
\hline Donor cytomegalovirus seropositive (no.) & $63.2 \%$ & $53.9 \%$ & $83.3 \%$ & .33 \\
\hline Donor ischemia time (min, mean $\pm \mathrm{SD}$ ) & $197 \pm 65$ & $224 \pm 56$ & $155 \pm 58$ & .01 \\
\hline \multicolumn{5}{|l|}{ Retransplant } \\
\hline Age at transplant $(y$, mean \pm SD) & $11.5 \pm 4.6$ & $10.6 \pm 3.9$ & $12.8 \pm 5.3$ & .25 \\
\hline Listing diagnosis rejection-related graft failure (no.) & $16(61 \%)$ & $6(40.0 \%)$ & $10(90.9 \%)$ & .014 \\
\hline Listing diagnosis cardiac allograft vasculopathy (no.) & $7(27 \%)$ & $6(40 \%)$ & $1(9.1 \%)$ & .18 \\
\hline Listing diagnosis other* (no.) & $3(11 \%)$ & $3(20 \%)$ & $0(0 \%)$ & .24 \\
\hline Donor ischemia (min, mean $\pm \mathrm{SD}$ ) & $221 \pm 63$ & $237 \pm 55$ & $195 \pm 71$ & .12 \\
\hline Ventilator time ( $\mathrm{d}$, median and range) & $1.5(1-8)$ & $2(1-3)$ & $1(1-8)$ & .14 \\
\hline Intensive care unit stay ( $\mathrm{d}$, median and range) & $5.5(3-44)$ & $6(3-9)$ & $5(3-44)$ & .25 \\
\hline Hospital stay (d, median and range) & $7(5-100)$ & $8(5-22)$ & $7(6-100)$ & .25 \\
\hline Early mortality (no.) & $2(8 \%)$ & $0(0 \%)$ & $2(18 \%)$ & .17 \\
\hline
\end{tabular}

*Three patients had severe tricuspid regurgitation and right ventricular dysfunction precluding initial or repeated attempts at tricuspid valve repair or replacement.

\section{Retransplant Outcomes}

Early survival after retransplant was $92 \%$. One patient died intraoperatively from aortic injury. A second patient arrested during anesthetic induction and died of severe brain injury. These deaths were of the first 2 patients in the series, and there were no early deaths since 1995 . There were 7 late deaths: sudden death with severe CAV at postmortem $(\mathrm{n}=4)$, sepsis $(\mathrm{n}=2)$, right ventricular failure with pulmonary hypertension $(\mathrm{n}=1)$, and acute rejection $(\mathrm{n}=1)$. Two infant group patients died late ( 0.7 and 11.9 years), and 5 pediatric group patients died late $(0.1,1.5,1.6,4.0$, and 9.4 years). No patient underwent a third transplant.

Mean retransplant follow-up for all patients was $4.0 \pm$ 4.1 years. Median survival after retransplant was 11.9 years. Estimated retransplant survivals at 1, 3, and 5 years were $83 \%, 74 \%$, and $67 \%$, respectively (Figure 1). Mean re-

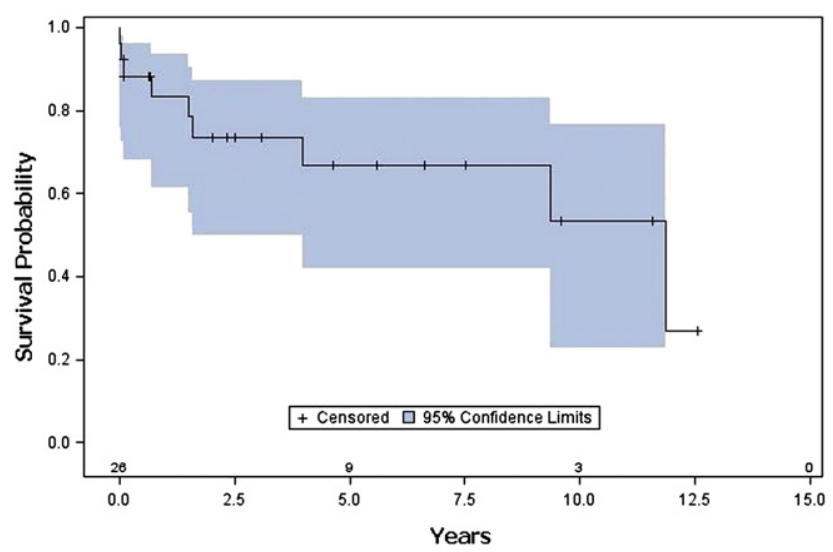

FIGURE 1. Kaplan-Meier estimated survival after retransplant for all patients. Median survival was 11.9 years. Patients at risk are listed above $\mathrm{x}$-axis. transplant follow-ups were $3.3 \pm 3.4$ years and $5.1 \pm 4.9$ years for the infant and pediatric groups, respectively. Median survivals were 11.9 years $(95 \%$ confidence interval [CI], 0.71-11.9 years) for the infant group and 4.0 years (95\% CI, 0.041 years to no upper limit) for the pediatric group $(P=.076)$. Five infant group patients had censored follow-up less than 1 year, whereas no pediatric group patients had censored follow-up before 5 years. More late deaths occurred in the pediatric group, but the number of infant group patients with censored short follow-up precluded comparison of late survival.

\section{Primary Graft Outcomes and Group Comparisons}

Median primary graft survival for all patients was 7.2 years. Median primary graft survival for the infant group was more than double that for the pediatric group (infant group: 10.7 years; $95 \%$ CI, 5.6-11.3 years; vs pediatric group: 3.9 years; $95 \% \mathrm{CI}, 2.1-5.3$ years; $P<.001$; Figure 2).

All indices of rejection were greater in the pediatric group (Figure 3). The mean number of rejection episodes in the infant group was $3.1 \pm 2.1$ (range, 0-6), compared with $4.8 \pm 2.5$ (range, 2-10) for the pediatric group $(P=.032)$. Every patient in the pediatric group had 2 or more rejection episodes. Two infant group patients had no rejection episodes before retransplant. Because the infant group graft survival was longer than that in the Pediatric Group, rejection rate as a function of total graft life was also assessed. The infant group mean rejection rate $(0.4 \pm$ 0.4 rejection episodes/y) was significantly less than that in the pediatric group $(1.5 \pm 1.1$ rejection episodes/y, $P=$.0024). The pediatric group had more rejection 


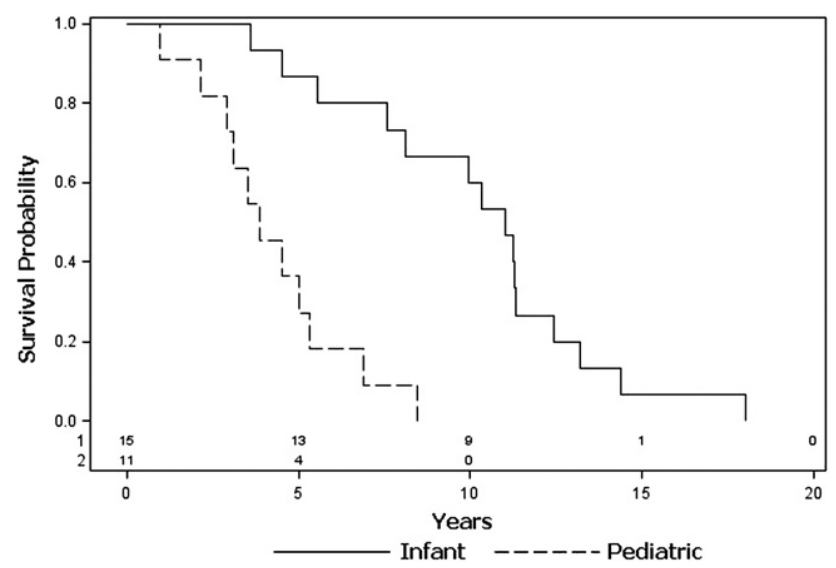

FIGURE 2. Kaplan-Meier estimated survival of primary transplant graft by group, with number of patients at risk in each group listed above $\mathrm{x}$-axis (infant group on top). Median survivals were 11.0 years (range 5.6-11.3 years) for infant group versus 3.9 years (range 2.1-5.3 years) for pediatric group $(P<.001)$.

episodes in the first year after primary transplant (infant group $0.53 \pm 0.99$ vs pediatric group $1.6 \pm 1.9$, $P=.0083$ ). According to protocol, patients in the infant group were managed with lower cyclosporine levels, and 11 of 15 were weaned to single-drug therapy. Recurrent rejection was the dominant retransplant listing diagnosis for 10 of 11 pediatric group patients $(91 \%)$, compared with 6 of 15 infant group patients $(40 \%)(P=.014$; Table 1$)$.

Table 2 presents the incidence and severity of primary graft CAV. Eleven of 15 infant group patients $(73 \%)$ had CAV, compared with 2 of 10 patients $(20 \%)$ in the pediatric group $(P=.032)$. CAV was the dominant retransplant listing diagnosis for 6 of 15 patients in the infant group (40\%), compared with 1 of 11 pediatric group patients $(P=.18)$. Putative risk factors for the development of CAV in pediatric recipients that were compared across groups included older recipient and donor ages, ${ }^{14}$ rejection episodes within

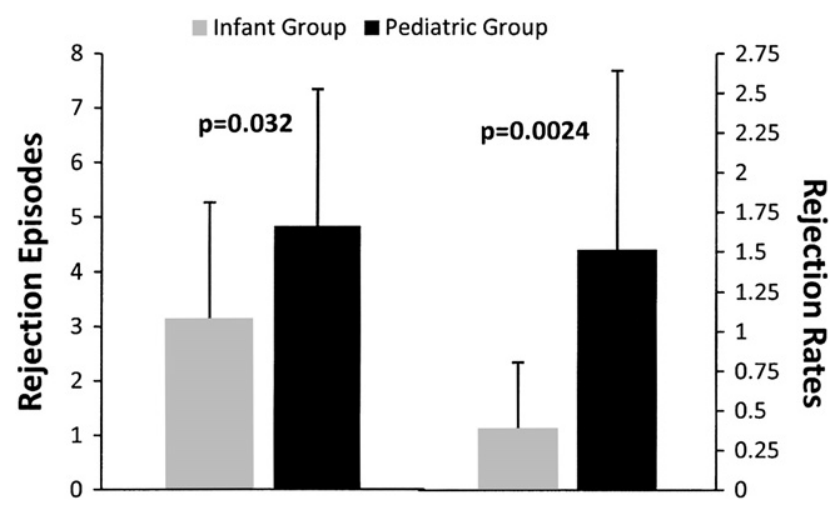

FIGURE 3. Comparison of rejection episodes and rejection rates by group.
TABLE 2. Incidence and classification of cardiac allograft vasculopathy

\begin{tabular}{lcc}
\hline Grade & Infant group $(\mathbf{n}=\mathbf{1 5})$ & Pediatric group $(\mathbf{n}=\mathbf{1 0} *)$ \\
\hline None & $4(27 \%)$ & $8(80 \%)$ \\
Mild & $3(20 \%)$ & $0(0 \%)$ \\
Moderate & $2(13 \%)$ & $0(0 \%)$ \\
Severe & $6(40 \%)$ & $2(20 \%)$ \\
Any & $11(73 \%)$ & $2(20 \%) \dagger$ \\
\hline *Angiogram missing for 1 patient. $\dagger P=.032$. &
\end{tabular}

*Angiogram missing for 1 patient. $\nmid P=.032$.

1 year of initial transplant, ${ }^{14}$ late $(>1$ year) rejection frequency and severity, ${ }^{20}$ short donor ischemia time $(<2$ hours), ${ }^{12}$ and positive recipient cytomegalovirus serostatus before first transplant. ${ }^{21}$ Cytomegalovirus serostatus was not different between groups (Table 1). The only differences between groups were older recipient and donor ages for the pediatric group (Table 1) and the previously delineated increased indices of rejection observed in the pediatric group. No putative risk factor for the development of CAV was increased in the infant group relative to the pediatric group other than the graft time at risk that resulted from the longer graft survival in the infant group. Notably, both patients in the infant group with no rejection episodes had severe CAV.

\section{DISCUSSION}

The graft half-life after primary heart transplant in pediatric patients currently approaches 14 years. ${ }^{12}$ Survival is significantly better than for adults, but pediatric graft survival remains limited. Retransplants now comprise $5 \%$ of all pediatric heart transplants performed. ${ }^{12}$ In centers with large numbers of transplant recipients, this incidence is probably higher. In the past 2 years, 7 of 38 pediatric heart transplants performed at our center $(18 \%)$ were retransplants. Retransplant is the only viable therapeutic option for graft failure, and our results substantiate that retransplant in pediatric patients is reasonable. As demonstrated by others, ${ }^{5,7,11}$ our retransplant 1-, 3-, and 5-year outcomes $(83 \%, 74 \%$, and $67 \%$, respectively) are similar to primary transplant outcomes $(85 \%, 78 \%$, and $75 \%$, respectively, $P=.40)$. All single-center reports, however, are limited in size and follow-up. ${ }^{5,7,11}$ Recent UNOS and International Society for Heart and Lung Transplantation registry reports clearly demonstrate worse 5-year outcomes for retransplant than for primary transplant, ${ }^{4,9,12}$ and we do not consider equivalence to primary transplant necessary to justify retransplant.

Although the mechanism is speculative, the plasticity of the immature infant immune system probably accounts for the survival advantage of primary infant transplant recipients relative to older recipients. Because infants have an advantage in adaptive immunity, our center and many others use less immunosuppression for these recipients, with 
outcomes superior to those of older patients. Previous studies on pediatric retransplant have not assessed whether age at first transplant impacts primary graft survival in patients undergoing retransplant, the etiology of primary graft failure in this subset, or retransplant graft survival. In our data set, primary graft survival for infant recipients who underwent retransplant was substantially better than that for patients who had undergone primary transplant at an older age. Because of the known graft survival advantage for infant recipients, ${ }^{12}$ the superior primary graft survival in our retransplant infant group was not unexpected. The difference in primary graft survival between age groups (nearly 3fold) was, however, more substantial than one would likely extrapolate from registry data. Whether this survival advantage extends to the second graft remains unanswered. Both study groups underwent retransplant at similar ages (Table 1), but underlying developmental differences in immunity and treatment histories may have affected second graft survival. We observed a trend toward better second graft survival in the infant group (median, 11.9 years vs 4.0 years in the pediatric group; $P=.076$ ), but our analysis was too limited by insufficient follow-up in the infant group to draw a valid conclusion. Longer follow-up may answer this question, but an analysis of registry data would be more appropriate.

When we compared our total retransplant experience with those of others, the relative etiologies of graft failure leading to listing for retransplant differed considerably. ${ }^{4-5,7,9,11} \mathrm{We}$ observed a lower incidence of $\mathrm{CAV}$ as the dominant diagnosis $(27 \%)$ and a higher incidence of rejection as the dominant diagnosis $(62 \%)$. In registry studies and previous pediatric single-center series, CAV was the leading etiology of graft failure, and rejection-related graft failure was less frequent. ${ }^{4-5,7,9,11-12}$ Several factors may explain this disparity. Patient management and recipient age spectrum may differ at our center. Second, determination of graft failure etiology when listing a patient for retransplant is sometimes subjective, and practices vary among centers. Many centers categorize a significant number of graft failures as nonspecific or other, and it is possible that many of these cases would be more definitively classified in our center. $^{4,7,9,12}$ Other centers may not distinguish a dominant etiology in patients with significant rejection history and concomitant CAV. ${ }^{11}$ We have endeavored to establish a dominant diagnosis for these patients. The choice between rejection and CAV as the dominant factor in patients with concomitant etiologies was made on the basis of clinical rejection history, serial biopsy findings, and serial coronary assessments. Third, the threshold for listing a patient for retransplant for $\mathrm{CAV}$ probably varies among centers. In our center, patients with $\mathrm{CAV}$ are not listed for retransplant until there is evidence of rapid progression of $\mathrm{CAV}$ or graft failure, manifesting as hemodynamic compromise, arrhythmia, or symptoms. A lower threshold at other centers would increase the incidence of graft failure from CAV relative to that at our center. It is well known that coronary arteriography lacks sensitivity in the detection of CAV until late in the course of disease ${ }^{20,22}$; however, the diagnosis of CAV, as with the other reports, was based on selective coronary arteriography. All patients in our series underwent angiographic assessment before listing for retransplant, and it is unlikely that the overall incidence of angiographically evident CAV was underestimated. Finally, all patients at our center with rejection-related etiology had a strong history of recurrent rejection when listed for retransplant.

We consider the most important findings of our study to be the differences in rejection and prevalence of angiographically evident CAV noted between the infant group and the older children coming to retransplant. International Society for Heart and Lung Transplantation registry and PHTS data indicate that the incidence of angiographically evident CAV is lower in younger recipients at any given time point through 8 years of follow-up. ${ }^{12,14}$ In addition, older recipient and donor ages at transplant are important risk factors for the development of $\mathrm{CAV}$ in pediatric recipients. ${ }^{14}$ Thus the high prevalence of CAV observed in our infant group relative to the older group ( $73 \%$ vs. $20 \%$, $P=0.032$ ) seems paradoxic. No reported patient CAV risk factors that we studied were increased in the infant group. The most likely reason for the age-related difference in CAV prevalence is therefore the difference in graft survival between groups (10.7 vs 3.9 years). All studies of CAV indicate that the risk of development of CAV at any age increases with time. Consequently, graft loss as a result of direct rejection-mediated myocardial injury likely occurred before angiographically evident CAV had time to develop in the older patients. Conversely, the longer primary graft life in infant recipients allowed sufficient time for angiographically evident CAV to develop before retransplant was required for other reasons. Importantly, severe CAV can occur in the absence of any history of rejection episodes, as occurred with 2 of our infant patients.

Because the risk of femoral vessel injury and occlusion is increased in infants who require lifelong surveillance, rejection surveillance at our center is primarily noninvasive during the first year after transplant in infants. Although first-year surveillance biopsy protocols differed between the groups, several factors suggest that this difference did not influence the increased indices of rejection observed in the pediatric group. First, our surveillance protocol used for infants was previously validated against endomyocardial biopsy in this age range. ${ }^{15}$ Second, the diagnosis of rejection at all ages was based on the combination of clinical, echocardiographic, and catheterization studies. Importantly, only 1 rejection episode in the pediatric group was diagnosed on the basis of a surveillance biopsy in isolation. Finally, if rejection episodes were under diagnosed early in the infant group because of less frequent use of surveillance 
biopsies, these missed rejections should have led to inadequate immune suppression and clinically apparent rejections or deaths in the infant group that were not, in fact, observed. Consequently, we believe that the reported differences observed in rejection parameters according to age at primary transplant are real.

The causes of CAV are not well understood, but both immune and nonimmune mechanisms are clearly involved. Treatment and prevention strategies for CAV in pediatric patients vary among centers, and although retransplant is the only option for patients with severe or symptomatic disease, medical management of CAV is without clear consensus. We have previously described our approach to the medical management of CAV in children. ${ }^{23}$

Although CAV was a leading cause of graft failure in the infant group in this study, the incidence of CAV in our entire infant primary recipient population was only $12 \%$. In a separate evaluation of CAV in our entire primary transplant population, the prevalences of CAV were similar between infant and pediatric recipients. ${ }^{24}$ The time to diagnosis of $\mathrm{CAV}$, however, was significantly longer in infant recipients than in pediatric recipients $(8.0 \pm 3.4$ years vs $5.4 \pm 3$ years, $P<.01)$. The severity of $\mathrm{CAV}$ at initial diagnosis was significantly worse in infant recipients than in pediatric recipients, and we observed a trend toward shorter time from diagnosis of CAV to death or retransplant in infant recipients $(0.9 \pm 0.9$ years vs $2.8 \pm 3.4$ years, $P=.06)$. Thus in our experience although CAV develops later in infant recipients, it is more virulent in infant recipients, and more than $50 \%$ of the infants with CAV diagnosed have undergone retransplant.

In addition to inherent limitations of retrospective studies, several factors limit this study. We considered only the subset of pediatric heart recipients with graft failure who underwent retransplant. Comparisons with the total population of patients undergoing primary transplant would be needed to delineate underlying factors that may have prompted the increased rejection-related graft failure and shorter primary graft life in the older retransplant patient group. Likewise, conclusions regarding the underlying causes of CAV cannot be made. Although this study represents the largest singlecenter experience and longest follow-up for pediatric retransplant to date, the patient numbers and relatively short follow-up limit its power. Finally, some findings in this study might have differed if patients at our center with graft failure who died without retransplant or underwent retransplant elsewhere had been included.

\section{CONCLUSIONS}

Our results confirm that heart retransplant is reasonable treatment for pediatric patients with late graft failure. Despite less aggressive immunosuppression, infant heart transplant recipients listed for retransplant had significantly longer primary graft survival and significantly lower indices of clinical rejection before retransplant than did children who were older at the time of primary transplant. The prevalence of primary graft $\mathrm{CAV}$ among infant recipients requiring retransplant was significantly higher than that among retransplant recipients who were older at primary transplant, despite more rejection in the latter group. Rejection-mediated injury likely resulted in more rapid graft failure in the older group before time-related factors that cause CAV could promote the development of CAV. Despite the primary graft survival advantage enjoyed by infant heart recipients, CAV is a major cause of graft loss in this age group.

\section{References}

1. Fortuna RS, Chinnock RE, Bailey LL. Heart transplantation among 233 infants during the first six months of life: the Loma Linda experience. Loma Linda Pediatric Heart Transplant Group. Clin Transpl. 1999;263-72.

2. Mitchell MB, Campbell DN, Clarke DR, Fullerton DA, Grover FL, Boucek MM, et al. Infant heart transplantation: improved intermediate results. J Thorac Cardiovasc Surg. 1998;1162:242-52.

3. Baum D, Bernstein D, Starnes VA, Oyer P, Pitlick P, Stinson E, et al. Pediatric heart transplantation at Stanford: results of a 15-year experience. Pediatrics. 1991;88:203-14.

4. Chin C, Naftel D, Pahl E, Shankel T, Clark ML, Gamberg P, et al. Cardiac retransplantation in pediatrics: a multi-institutional study. J Heart Lung Transplant. 2006;25:1420-4.

5. Dearani JA, Razzouk AJ, Gundry SR, Chinnock RE, Larsen RL, del Rio MJ, et al. Pediatric cardiac retransplantation: intermediate-term results. Ann Thorac Surg. 2001;71:66-70.

6. Johnson MR, Aaronson KD, Canter CE, Kirklin JK, Mancini DM, Mehra MR, et al. Heart retransplantation. Am J Transplant. 2007;7:2075-81.

7. Kanter KR, Vincent RN, Berg AM, Mahle WT, Forbess JM, Kirshbom PM. Cardiac retransplantation in children. Ann Thorac Surg. 2004;78:644-9.

8. Mahle WT. Cardiac retransplantation in children. Pediatr Transplant. 2008;12: 274-80.

9. Mahle WT, Vincent RN, Kanter KR. Cardiac retransplantation in childhood: analysis of data from the United Network for Organ Sharing. J Thorac Cardiovasc Surg. 2005;130:542-6.

10. Michler RE, Edwards NM, Hsu D, Bernstein D, Fricker FJ, Miller J, et al. Pediatric retransplantation. J Heart Lung Transplant. 1993;12(6 Pt 2):S319-27.

11. Richmond ME, Addonizio LJ, Hsu DT, Mital SR, Mosca RS, Quaegebeur JM, et al. Cardiac retransplantation in high risk pediatric patients. Pediatr Transplant. 2007;11:615-23.

12. Kirk R, Edwards LB, Aurora P, Taylor DO, Christie JD, Dobbels F, et al. Registry of the International Society for Heart and Lung Transplantation: Twelfth Official Pediatric Heart Transplantation Report-2009. J Heart Lung Transplant. 2009; 28:993-1006.

13. The International Society for Heart \& Lung Transplantation [Internet]. Addison (TX): The Society; c2003 [cited 2010 Apr 30]. Registries. Available from: http:// www.ishlt.org/registries/

14. Pahl E, Naftel DC, Kuhn MA, Shaddy RE, Morrow WR, Canter CE, et al. The impact and outcome of transplant coronary artery disease in a pediatric population: a 9-year multi-institutional study. J Heart Lung Transplant. 2005;24: $645-51$

15. Boucek MM, Mathis CM, Boucek RJ Jr, Hodgkin DD, Kanakriyeh MS, McCormack J, et al. Prospective evaluation of echocardiography for primary rejection surveillance after infant heart transplantation: comparison with endomyocardial biopsy. J Heart Lung Transplant. 1994;13:66-73.

16. Chin C, Naftel DC, Singh TP, Blume ED, Luikart H, Bernstein D, et al. Risk factors for recurrent rejection in pediatric heart transplantation: a multicenter experience. J Heart Lung Transplant. 2004;23:178-85.

17. Kirklin JK, Brown RN, Huang ST, Naftel DC, Hubbard SM, Rayburn BK, et al. Rejection with hemodynamic compromise: objective evidence for efficacy of photopheresis. J Heart Lung Transplant. 2006;25:283-8.

18. Webber SA, Naftel DC, Parker J, Mulla N, Balfour I, Kirklin JK, et al. Late rejection episodes more than 1 year after pediatric heart transplantation: risk factors and outcomes. J Heart Lung Transplant. 2003;22:869-75. 
19. Costanzo MR, Naftel DC, Pritzker MR, Heilman JK 3rd, Boehmer JP, Brozena SC, et al. Heart transplant coronary artery disease detected by coronary angiography: a multiinstitutional study of preoperative donor and recipient risk factors. Cardiac Transplant Research Database. J Heart Lung Transplant. 1998; 17:744-53.

20. Mulla NF, Johnston JK, Vander Dussen L, Beeson WL, Chinnock RE, Bailey LL, et al. Late rejection is a predictor of transplant coronary artery disease in children. J Am Coll Cardiol. 2001;37:243-50.

21. Hussain T, Burch M, Fenton MJ, Whitmore PM, Rees P, Elliott M, et al. Positive pretransplantation cytomegalovirus serology is a risk factor for cardiac allograft vasculopathy in children. Circulation. 2007;115:1798-805.

22. Kuhn MA, Jutzy KR, Deming DD, Cephus CE, Chinnock RE, Johnston J, et al. The medium-term findings in coronary arteries by intravascular ultrasound in infants and children after heart transplantation. J Am Coll Cardiol. 2000;36: 250-4.

23. Pietra B, Boucek M. Coronary artery vasculopathy in pediatric cardiac transplant patients: the therapeutic potential of immunomodulators. Paediatr Drugs. 2003; 5:513-24.

24. Tissot C, Pietra BA, Ivy DD, Campbell DN, Mitchell MB, Diamond BA, et al. Transplant coronary artery disease is diagnosed later and is more severe in pediatric heart recipients transplanted during infancy [abstract]. Cardiol Young. 2009;20(Suppl 2):S24.

\section{Discussion}

Dr Jonathan Chen (New York, NY). I would like to congratulate Dr Karamichalis and colleagues from the Denver Children's Hospital on an impressive series from one of the leading centers for pediatric heart transplantation in North America.

While survival following transplantation continues to improve, regrettably a sizable minority of these recipients will require retransplantation and, in this way, transplantation is for many children a palliative procedure that merely postpones an inevitable graft replacement anywhere from several years to several decades following the primary operation.

Our findings at Columbia have mirrored those of Denver, suggesting that the survival outcomes following retransplantation are now comparable to primary transplantation in well-selected candidates. The report today seeks to further characterize this subcohort of retransplant candidates with a particular interest on the age at their primary procedure.

Having had, thank you, the benefit to review your data prior to this presentation, I have 4 questions for the authors.

The first is: Were there any children with graft failure who were not deemed retransplant candidates during your study period and thus did not make it into this study?

Dr Karamichalis. We had patients who were listed on the waiting list for retransplantation. We had 10 patients who were listed for retransplantation: 5 of them died on the transplant list, 2 of them were transferred to another facility, and 3 of them were removed from the list because their condition improved.

Dr Chen. Second, how was the decision made to divide these patients into groups that were greater than and less than 1 year of age? Inferences from ABO-incompatible strategies would suggest that the time of so-called immunologic privilege can extend as far as 14 months or longer.

Dr Karamichalis. Because of the known immaturity of the infant immune system, we felt that the infant recipient population would have an advantage over anybody over 1-year-old, so we divided the groups into less than 1 year or over 1 year. The ISHLT database registry divides these patients into more than 2 sub- groups; however, because of our small group, we decided to draw the line between infants and older than 1 .

Dr Chen. Third, I'm somewhat perplexed still at the finding that there is more transplant coronary disease but fewer incidences of rejection in your infant subcohort. And I wonder whether some of this could be due to the differences in surveillance that you describe with respect to your age-based protocols that dictate the timing of the endomyocardial biopsies.

Dr Karamichalis. The data concerning the short follow-up of those 5 patients corresponds to the time after retransplantation. All patients that had their first transplant and made it to retransplantation had surveillance angiograms at the first anniversary of their transplant and subsequently every other year. If there was evidence of CAV, then they would receive more frequent surveillance for $\mathrm{CAV}$ and treatments.

At the time of catheterization, all patients also had endomyocardial biopsies. So given the median graft survival of both the infant and the older group, the older being 3.9 years, then all of those patients should have at least had 2 or 3 endomyocardial biopsies and at least 3 angiograms.

Dr Chen. Do you know if it's the practice to treat episodes of transplant coronary disease in the absence of rejection? So could it be that the coronary disease was detected in the infants and they were treated before there was biopsy-proven rejection and thus the lower incidence of rejection?

Dr Karamichalis. Once CAV is diagnosed in these patients at our center, they get treated. And the strategy that we use, includes statins or antilipid agents, we also use MMF and rapamycin, an antiproliferative agent, to decelerate the progression of CAV in these patients. So any evidence or any signs of CAV, no matter how severe or mild they might be, they get treated. The treatment escalates based on the severity of the CAV.

Dr Chen. Finally, is it possible that the decreased graft survival and increased rejection in your group 2 was related to medication noncompliance, given that the fact that the average age of this group was 9 , plus or minus 6 years, at their primary transplant, which would render many of them medically recalcitrant adolescents at the time of their initial post-transplant years.

Dr Karamichalis. I think that's an excellent point and it's definitely something worth mentioning. In the older population, there may be a compliance issue of which we are not aware. Even though we discussed it, we haven't really searched specifically for noncompliance in these patients, but it's definitely something that is well known to cause rejection in patients who don't take their medications.

Dr Chen. Thanks. These are very provocative and very interesting data. I'm very interested in how these findings play out as you follow these patients postretransplant.

Dr Carl Backer (Chicago, Ill). John, congratulations on a very nice analysis. Clearly, this is a huge amount of work.

I have a question. Given the data that you have on retransplantation, I'd like to ask your opinion about 2 high-risk groups. The first is the patient who has had their first transplant, doesn't come off bypass because they have right ventricular dysfunction, and now you're contemplating whether or not you should relist them for another transplant. Did you have any patients like that in your series and what are your thoughts about that population group?

Dr Karamichalis. Thank you for your comments and your question. 
No, we did not have any relisting of early graft failure. One patient retransplanted was more than a year after his primary transplant. Everybody else got retransplanted at 2 or more years after their primary transplant. So I do not believe we have anybody that was relisted following primary transplantation with failure to come off bypass.

Dr Backer. The second question is: What do you think about a third-time transplant? Do you have any patients who you've transplanted a third time and what's your opinion about that?
Dr Karamichalis. I'm aware of 1 patient who was offered third-time retransplantation. That patient refused to be relisted and died subsequently of PTLD, which was unrelated to CAV or rejection. I know other centers have reported third-time retransplantations. I guess it would be a collective decision between everybody involved. And, if somebody makes a compelling case, then a third transplant should be offered but it would only be on a case-to-case basis. 